\title{
A COMPARISON OF ALGEBRAIC, METRIC, AND LATTICE BETWEENNESS
}

\author{
M. F. SMILEY
}

Introduction. We propose to investigate here the consequences of the identity of each pair chosen from three important generalizations of the relation of betweenness on a line, namely, algebraic betweenness $\left[1\right.$, p. 27], ${ }^{1}$ metric betweenness [3, p. 36], and lattice betweenness $[7$, Part II]. We shall also find an interpretation of metric betweenness in the Banach space of all continuous functions defined on the interval $0 \leqq t \leqq 1$ which can be used to establish the fact that this relation satisfies no strong four or five point transitivity [7, Part I] except $t_{1}$ and $t_{2}$.

We note first that algebraic betweenness implies metric betweenness and lattice betweenness. We find that algebraic betweenness and metric betweenness coincide in a seminormed real vector space ${ }^{2}$ if and only if it is strictly convex in the sense of Clarkson [4, p. 404]. We then show that the coincidence of metric and lattice betweenness in a semimetric space $[3$, p. 38] which is also a lattice $[2$, p. 16] leads to a system which is a metric lattice (in the sense of G. Birkhoff $[2$, p. 41]). It follows that a complete seminormed real vector lattice is equivalent to an $(L)$-space [6] if and only if its metric and lattice betweenness relations are identical. Finally, we prove that algebraic and lattice betweenness coincide in a real vector lattice if and only if it is equivalent to the system of all real numbers. We conclude by giving the interpretation of metric betweenness in the space ${ }^{3} C[0,1]$. 1942.

Presented to the Society., September 10, 1942; received by the editors March 27,

${ }^{1}$ References to the bibliography at the end of the paper will be in brackets.

${ }^{2}$ We shall use these terms as follows. A seminormed real vector space is a vector space over the field of all real numbers together with a real non-negative single-valued function $\|a\|$, called the "norm of $a$," satisfying (i) $\|\lambda a\|=|\lambda|\|a\|$, and (ii) $\|a\|=0$ if and only if $a=0$. A normed real vector space satisfies in addition (iii) $\|a\|+\|b\| \geqq\|a+b\|$. A real vector lattice is a vector space over the field of all real numbers which is also a lattice $[2$, p. 16] with respect to a partial ordering relation " $\geqq$ " such that (i) $a \geqq b$ and $\lambda \geqq 0$ implies $\lambda a \geqq \lambda b$, and (ii) $a \geqq b$ implies $a+c \geqq b+c$ for every c. A (semi)normed real vector lattice is a real vector lattice which is also a (semi)normed real vector space; it is complete if evey fundamental sequence has a limit. A complete normed real vector space is usually called a (real) Banach space.

${ }^{3}$ The notation $C[0,1]$ (sometimes simply $C$ ) is currently used to designate the space described in the concluding sentence of the preceding paragraph. 
1. The three betweenness relations. We shall be interested in the following relations:

$$
\begin{aligned}
& (a b c)_{A} \equiv b=\lambda a+(1-\lambda) c, \quad \lambda \text { a real number, } 0 \leqq \lambda \leqq 1, \\
& (a b c)_{M} \equiv \delta(a, b)+\delta(b, c)=\delta(a, c), \\
& (a b c)_{L} \equiv(a \cap b) \cup(b \cap c)=b=(a \cup b) \cap(b \cup c),
\end{aligned}
$$

to which we shall refer as $R_{A}$ (algebraic betweenness), $R_{M}$ (metric betweenness), and $R_{L}$ (lattice betweenness). The relation $R_{A}$ applies to a real vector space, $R_{M}$ to a semimetric space, and $R_{L}$ to a lattice. Our purpose is to find those systems to which each two of these three relations apply and have the same meaning.

The following three lemmas are known or evident.

LEMMA 1. If $S$ is a seminormed real vector space, then we have $(a b c)_{M}$ if and only if $\|a-b\|+\|b-c\|=\|a-c\|$.

LEMMA 2. If $S$ is a distributive lattice, then $\left(\begin{array}{lll}a & b & c\end{array}\right)_{L}$ if and only if $a \cap c \leqq b \leqq a \cup c$.

Proof. This is Lemma 9.1 of [7].

LEMMA 3. If $S$ is a seminormed real vector space, then $\left(\begin{array}{lll}a & b\end{array}\right)_{A}$ $\rightarrow(a b c)_{M}$ for every $a, b, c \in S$.

LEMMA 4. If $S$ is a real vector lattice, then $(a b c)_{A} \rightarrow(a b c)_{L}$ for every $a, b, c \in S$.

Proof. It is well known that a vector lattice is distributive [2, p. 108], and hence by Lemma 2 it will suffice to show that $a \cap c \leqq b \leqq a \cup_{c}$ is a consequence of $(a b c)_{A}$. We have the equations

$$
\begin{aligned}
(a \cup c)-b=(a-b) \cup(c-b) & =(1-\lambda)(a-c) \cup-\lambda(a-c) \\
& =((a-c) \cup 0)-\lambda(a-c) .
\end{aligned}
$$

Note that $(a-c) \cup 0 \geqq \lambda((a-c) \cup 0)$, since $\lambda \leqq 1$; and that $\lambda((a-c) \cup 0)$ $=\lambda(a-c) \cup 0 \geqq \lambda(a-c)$, since $\lambda \geqq 0$. Hence $(a \cup c)-b \geqq 0$, that is, $a \cup c \geqq b$. The other inequality is dual.

2. Coincident metric and algebraic betweenness. Clarkson $[4, \mathrm{p}$. 404] calls a normed real vector space strictly convex if the equality $\|a\|+\|b\|=\|a+b\|$ for nonzero $a$ and $b$ implies that $a=\mu b$ for some $\mu>0$. The following theorem then tells us when metric and algebraic betweenness coincide.

THEOREM 1. A seminormed real vector space $S$ is strictly convex if and only if algebraic and metric betweenness coincide in $S$. 
Proof. Let $S$ be a strictly convex seminormed real vector space. By Lemma 3, we need only show that $(a b c)_{M} \rightarrow(a b c)_{A}$ for every $a, b, c \in S$. We may clearly suppose that $a-b$ and $b-c$ are nonzero. But then the strict convexity of $S$ implies that $a-b=\mu(b-c)$ for some $\mu>0$. The choice $\lambda=1 / 1+\mu$ is effective for $(a b c)_{A}$. Conversely, if $(a b c)_{M} \rightarrow(a b c)_{A}$ for every $a, b, c \in S$, the equality $\|a\|+\|b\|=\|a+b\|$ for nonzero $a$ and $b$ implies $(a 0-b)_{A}$, and consequently that $0=\lambda a-(1-\lambda) b$ for real $\lambda$ between 0 and 1 . The choice $\mu=(1-\lambda) / \lambda$ is then effective in the definition of the strict convexity of $S$. The proof is complete.

Remark 1. Clarkson [4, p. 413] also shows that every separable Banach space may be renormed to secure strict convexity.

3. Coincident metric and lattice betweenness. Our definition of lattice betweenness arose as a generalization of the metric betweenness of a metric lattice $[2$, p. $41 ; 5,9]$. We shall show in this section that, conversely, a semimetric space which is also a lattice has identical lattice and metric betweenness relations only if it is a metric lattice. ${ }^{4} \mathrm{We}$ apply this result to give a characterization of $(L)$-spaces in terms of betweenness relations. ${ }^{5}$

Let us consider a semimetric space $M$ with distance function $\delta(a, b)$ which is also a lattice and in which lattice betweenness and metric betweenness coincide.

Lemma 5. If $a, b, c \in M$ and $a \leqq b \leqq c$, then $(a b c)_{M}$. If $a, b \in M$ then $(a a \cup b b)_{M}$ and $(a a \cap b b)_{M}$.

Proof. This is clear from Lemma 8.1 of [7].

Lemma 6. If $a, b \in M$, then $\delta(a, b)=\delta(a \cup b, a \cap b)$, and $\delta(a \cap b, b)$ $=\delta(a, a \cup b)$.

Proof. Consider two elements $a, b \in M$. By Lemma 5 we have the relations $(a a \cup b b)_{M},(a a \cap b b)_{M},(a \cap b b a \cup b)_{M}$, and $(a \cap b a a \cup b)_{M}$. Hence we have the following equations:

4 It should be noted that the results of $\$ 2$ remain valid if we merely require the values of $\delta(a, b)$ to be in a real vector lattice, provided the terms modular functional, metric lattice, and so on, are given proper interpretations.

${ }^{5} \mathrm{G}$. Birkhoff's remark that $m[a] \equiv\left\|a_{+}\right\|-\left\|a_{-}\right\|$is a sharply positive modular functional in an $(L)$-space (for proof, see [8]) aided the author materially in securing the present form of Theorem 2. The functional $m[a]$ is also linear. Its homogeneity follows readily from that of $\|a\|$, while its additivity is a simple consequence of the identity $m[a]-m[b]=\|a-b\|$ for $a \geqq b$. When the condition (IX) of Kakutani [6] holds, or, equivalently, when $m[a \cup b]-m[a \cap b]=\|a-b\|$, we find that $m[a]$ is the value of the Lebesgue-Stieltjes integral of the function corresponding to $a$. 
$\delta(a, a \cup b)+\delta(a \cup b, b)=\delta(a, b)=\delta(a, a \cap b)+\delta(a \cap b, b)$, $\delta(a \cap b, b)+\delta(b, a \cup b)=\delta(a \cap b, a \cup b)=\delta(a \cap b, a)+\delta(a, a \cup b)$.

Subtracting these equations we see that

$$
\begin{aligned}
\delta(a, a \cup b)-\delta(a \cap b, b) & =\delta(a, b)-\delta(a \cup b, a \cap b) \\
& =\delta(a \cap b, b)-\delta(a, a \cup b) .
\end{aligned}
$$

It follows that the relations stated in the lemma hold.

REMARK 2. Note that the first result of Lemma 5 together with the results of Lemma 6 imply that the second result of Lemma 5 is valid. This is clear since $\delta(a, b)=\delta(a \cup b, a \cap b)=\delta(a \cup b, a)+\delta(a, a \cap b)$ $=\delta(a, a \cap b)+\delta(a \cap b, b)$, and dually.

\section{LEMMA 7. The lattice $M$ is modular.}

Proof. We base our proof on the fact that if $M$ fails to be modular then it contains as a sublattice the simplest non-modular lattice [2, p. 34]. Let the elements of this sublattice be $O, a, b, c, I$, with $I$ the greatest, $O$ the least element, and $b>c$. Since $O=a \cap c$ and $O<a<I$, Lemma 5 gives $\delta(O, c)=\delta(a, I)$. Since $O<c<b<I$, Lemma 5 and this result gives $\delta(a, I)+\delta(c, b)+\delta(b, I)=\delta(O, I)$. Again, since $I=a \cup b$, we have $\delta(O, I)+\delta(c, b)=\delta(O, I)$, and consequently $\delta(b, c)=0$, contrary to $b>c$. Hence $M$ cannot fail to be modular.

LEMmA 8. If $a, b, c \in M$ and $a \geqq b$, then

$$
\delta(a \cup c, b \cup c)+\delta(a \cap c, b \cap c)=\delta(a, b) .
$$

Proof. Consider elements $a, b, c \in M$ with $a \geqq b$. By Lemma 6 we have

$$
\delta(a \cup c, b \cup c)=\delta(a \cup b \cup c, b \cup c)=\delta(a, a \cap(b \cup c)) .
$$

Noting that $a \geqq a \cap(b \cup c) \geqq b$, we find from Lemma 5 that

$$
\delta(a \cup c, b \cup c)=\delta(a, b)-\delta(b, a \cap(b \cup c)) .
$$

Using Lemmas 7 and 6 we have

$$
\begin{aligned}
\delta(a \cup c, b \cup c) & =\delta(a, b)-\delta(b, b \cup(a \cap c)) \\
& =\delta(a, b)-\delta(a \cap c, b \cap a \cap c) .
\end{aligned}
$$

Since $a \geqq b$, this proves the lemma.

LEMMA 9. If $a^{*} \in M$ the functional $m[a] \equiv \delta\left(a \cup a^{*}, a^{*}\right)-\delta\left(a^{*}, a \cap a^{*}\right)$ is a sharply positive modular functional and $\delta(a, b)=m[a \cup b]-m[a \cap b]$ for every $a, b \in M$. 
Proof. From Lemma 6 and the fact that $\delta(a, b)>0$ for $a \neq b$, it will suffice to show that

$$
\delta(a, b)=m[a]-m[b]
$$

for every $a, b \in M$ such that $a \geqq b$. Hence consider elements $a, b \in M$ for which $a \geqq b$. Lemma 8 yields the equation

$$
\delta\left(a \cup a^{*}, b \cup a^{*}\right)+\delta\left(a \cap a^{*}, b \cap a^{*}\right)=\delta(a, b) .
$$

Since $a \cup a^{*} \geqq b \cup a^{*} \geqq a^{*}$, and dually, Lemma 5 yields the following equations

$$
\begin{aligned}
& \delta\left(a \cup a^{*}, a^{*}\right)-\delta\left(b \cup a^{*}, a^{*}\right)=\delta\left(a \cup a^{*}, b \cup a^{*}\right), \\
& \delta\left(a^{*}, b \cap a^{*}\right)-\delta\left(a^{*}, a \cap a^{*}\right)=\delta\left(a \cap a^{*}, b \cap a^{*}\right) .
\end{aligned}
$$

Adding these equations and using the equation (2) we obtain (1) from the definition of the functional $m[a]$. The proof is complete.

REMARK 3. The validity of Lemmas 7-9 depends only on Lemma 6 and the first result of Lemma 5.

Lemma 10. The distance function $\delta(a, b)$ is a metric for $M$.

Proof. This follows from Lemma 9 and Theorem 3.10 of [2].

THEOREM 2. If $S$ is a semimetric space with distance function $\rho(a, b)$ which is also a lattice, then metric betweenness and lattice betweenness coincide in $S$ if and only if:

(i) For every $a, b, c \in S$ the inequalities $a \leqq b \leqq c$ imply that $(a b c)_{M}$.

(ii) For every $a, b \in S, \rho(a, b)=\rho(a \cup b, a \cap b)$ and $\rho(a, a \cup b)$ $=\rho(b, a \cap b)$.

The conditions (i) and (ii) hold if and only if for each $a^{*} \in M$ the functional $m[a] \equiv \rho\left(a \cup a^{*}, a^{*}\right)-\rho\left(a^{*}, a \cap a^{*}\right)$ is a sharply positive modular functional and $S$ is a. metric lattice with metric $\rho(a, b)$ $=m[a \cup b]-m[a \cap b]$.

Proof. This is clear from Lemmas 5-9, Remarks 2 and 3, and Theorem 10.1 of [7].

We also have a specialization of Theorem 2.

TheOREM 3. A complete seminormed real vector lattice $S$ is equivalent to an $(L)$-space if and only if lattice and metric betweenness coincide in $S$.

Proof. Let $S$ be a complete seminormed real vector lattice in which lattice and metric betweenness coincide. By Theorem 2, Lemma 6, and Lemma 10, $S$ is a complete normed real vector lattice satisfying the conditions, 
(VIII) if $a, b \in S$ and $a \geqq 0, b \geqq 0$, then $\|a\|+\|b\|=\|a+b\|$;

(IX) if $a, b \in S$ and $a \cap b=0$, then $\|a+b\|=\|a-b\|$;

of Kakutani [6]. Kakutani's fundamental Theorem 7 [6] together with the fact that his condition (V) is not needed (in his Theorem 7) [8] now shows that $S$ is equivalent to an $(L)$-space.

Conversely, if $S$ is equivalent to an $(L)$-space, it is easy to verify the conditions (i) and (ii) of Theorem 2. Hence lattice betweenness and metric betweenness coincide in $S$. The proof is complete.

Remark 4. We may restate Theorem 3 as follows. A complete seminormed real vector lattice is equivalent to an $(L)$-space if and only if it is a metric lattice.

4. Coincident lattice and algebraic betweenness. We include the following theorem for the sake of completeness.

Theorem 4. If $S$ is a real vector lattice, then lattice betweenness and algebraic betweenness coincide in $S$ if and only if the dimension of $S$ (as a vector space) is one.

Proof. We need only show that $\left(\begin{array}{lll}a & b & c\end{array}\right)_{L} \rightarrow\left(\begin{array}{lll}a & b & c\end{array}\right)_{A}$ for every $a, b, c \in S$ implies that $S$ is one-dimensional. It is easy to verify that $R_{A}$ has the fundamental transitivity $t_{3}$ [7]. By Theorem 9.7 of [7] every pair of elements of $S$ are comparable by " $\geqq$." If, then, there were two linearly independent elements $a, b \in S$, we should have (by symmetry) $a>b>0$ or $a>0>b$; and hence [7, Lemma 8.1]

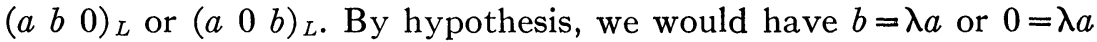
$+(1-\lambda) b$, which clearly contradicts the linear independence of $a$ and $b$. Thus $S$ is one-dimensional.

5. Metric betweenness in the space $C[0,1]$. The question of whether or not the metric betweenness of a normed real vector lattice possesses any more than the required transitivities (that is, $(\alpha),(\beta), t_{1}$, and $t_{2}$ of [7]) can be settled by the space $C[0,1]$. We give in this section an interpretation of $R_{M}$ in $C[0,1]$ and leave to the reader the construction of simple examples which show that this relation has only the required transitivities. This result should be contrasted with the result of Clarkson mentioned in Remark 1.

LEMMA 11 . In the space $C[0,1]$ the relation $(a 0 b)_{M}$ holds if and only if there is a point $t_{0}$ on $[0,1]$ at which each of the functions $|a(t)|$, $|b(t)|$, and $|a(t)-b(t)|$ attains its maximum value and the relation $\left(a\left(t_{0}\right) 0 b\left(t_{0}\right)\right)_{M}$ holds.

Proof. Suppose that the relation $(a 0 b)_{M}$ holds in $C[0,1]$. We may clearly assume that $a$ and $b$ are nonzero. Let $t_{0}$ be a point on $[0,1]$ at 
which the function $|a(t)-b(t)|$ attains its maximum value. Then we have

$$
\begin{aligned}
\|a\|+\|b\| & =\|a-b\|=\left|a\left(t_{0}\right)-b\left(t_{0}\right)\right| \\
& \leqq\left|a\left(t_{0}\right)\right|+\left|b\left(t_{0}\right)\right| \leqq\|a\|+\|b\| .
\end{aligned}
$$

It follows that $\left|a\left(t_{0}\right)\right|+\left|b\left(t_{0}\right)\right|=\|a\|+\|b\|$ and that each of the functions $|a(t)|$ and $|b(t)|$ attains its maximum at $t_{0}$. Consequently neither $a\left(t_{0}\right)$ nor $b\left(t_{0}\right)$ can be zero. If they should both have the same sign we would find that $\left|a\left(t_{0}\right)-b\left(t_{0}\right)\right|<\left|a\left(t_{0}\right)\right|+\left|b\left(t_{0}\right)\right|$. This is impossible, and we conclude that the relation $\left(a\left(t_{0}\right) 0 b\left(t_{0}\right)\right)_{M}$ holds.

Conversely, if there is a point $t_{0}$ on $[0,1]$ at which each of the functions $|a(t)|,|b(t)|$, and $|a(t)-b(t)|$ attains its maximum value and the relation $\left(a\left(t_{0}\right) \quad 0 \quad b\left(t_{0}\right)\right)_{M}$ holds, we see that

$$
\|a-b\|=\left|a\left(t_{0}\right)-b\left(t_{0}\right)\right|=\left|a\left(t_{0}\right)\right|+\left|b\left(t_{0}\right)\right|=\|a\|+\|b\| .
$$

The proof is complete.

Theorem 5 . In the space $C[0,1]$ the relation $(a b c)_{M}$ holds if and only if there is a point $t_{0}$ on $[0,1]$ at which each of the functions $|a(t)-b(t)|,|b(t)-c(t)|$, and $|a(t)-c(t)|$ attains its maximum value and the relation $\left(a\left(t_{0}\right) b\left(t_{0}\right) c\left(t_{0}\right)\right)_{M}$ holds.

Proof. This is an immediate consequence of Lemma 11 and the fact that $\left(\begin{array}{llll}a & b & c\end{array}\right)_{M}$ if and only if $(a+x b+x \quad c+x)_{M}$ for every $a, b, c, x \in C[0,1]$.

\section{BIBLIOGRAPHY}

1. S. Banach, Théorie des opérations linéaires, Warsaw, 1932.

2. G. Birkhoff, Lattice theory, Amer. Math. Soc. Colloquium Publications vol. 25, 1940.

3. L. M. Blumenthal, Distance geometries, University of Missouri Studies vol. 12 (1938) no. 2.

4. J. A. Clarkson, Uniformly convex spaces, Trans. Amer. Math. Soc. vol. 40 (1936) pp. 396-414.

5. V. Glivenko, Géométrie des systèmes de choses normées, Amer. J. Math. vol. 58 (1936) pp. 799-828.

6. S. Kakutani, Concrete representation of abstract $(L)$-spaces and the mean ergodic theorem, Ann. of Math. (2) vol. 42 (1941) pp. 523-537.

7. Everett Pitcher and M. F. Smiley, Transitivities of betweenness, Trans. Amer. Math. Soc. vol. 52 (1942) pp. 95-114.

8. M. F. Smiley, A remark on $S$. Kakutani's characterization of $(L)$-spaces, Ann. of Math. (2) vol. 43 (1942) pp. 528-529.

9. L. R. Wilcox and M.F.Smiley, Metric lattices, Ann. of Math. (2) vol. 40 (1939) pp. 309-327.

LEHIGH UNIVERSITY 